\title{
Digitally Continuous Multivalued Functions
}

\author{
Carmen Escribano, Antonio Giraldo, ${ }^{\star}$ and María Asunción Sastre \\ Departamento de Matemática Aplicada, Facultad de Informática \\ Universidad Politécnica, Campus de Montegancedo \\ Boadilla del Monte, 28660 Madrid, Spain
}

\begin{abstract}
We introduce in this paper a notion of continuity in digital spaces which extends the usual notion of digital continuity. Our approach uses multivalued maps. We show how the multivalued approach provides a better framework to define topological notions, like retractions, in a far more realistic way than by using just single-valued digitally continuous functions. In particular, we characterize the deletion of simple points, one of the most important processing operations in digital topology, as a particular kind of retraction.
\end{abstract}

Keywords: Digital space, continuous function, simple point, retraction.

\section{Introduction}

The notion of continuous function is the fundamental concept in the study of topological spaces. Therefore it should play also an important role in Digital Topology.

There have been some attempts to define a reasonable notion of continuous function in digital spaces. The first one goes back to A. Rosenfeld [13] in 1986. He defined continuous function in a similar way as it is done for continuous maps in $\mathbb{R}^{n}$. It turned out that continuous functions agreed with functions taking 4adjacent points into 4-adjacent points. He proved, amongst other results, that a function between digital spaces is continuous if and only it takes connected sets into connected sets.

More results related with this type of continuity were proved by L. Boxer in 1] and, more recently in 234. In these papers, he introduces such notions as homeomorphism, retracts and homotopies for digitally continuous functions, applying these notions to define a digital fundamental group, digital homotopies and to compute the fundamental group of sphere-like digital images. However, as he recognizes in 3], there are some limitations with the homotopy equivalences he get. For example, while all simple closed curves are homeomorphic and hence homotopically equivalent with respect to the Euclidean topology, in the digital case two simple closed curves can be homotopically equivalent only if they have the same cardinality.

A different approach was suggested by V. Kovalevsky in 11, using multivalued maps. This seems reasonable, since an expansion as $f(x)=2 x$ must take 1

\footnotetext{
* Partially supported by DGES.
} 
pixel to 2 pixels if the image of an interval has still to be connected. He calls a multivalued function continuous if the pre-image of an open set is open. He considers, however, that another important class of multivalued functions is what he calls "connectivity preserving mappings". By its proper definition, the image of a point by a connectivity preserving mapping is a connected set. This is not required for merely continuous functions. He finally asserts that the substitutes for continuous functions in finite spaces are the simple connectivity preserving maps, where a connectivity preserving map $f$ is simple if for any $x$ such that $f(x)$ has more than 1 element then $f^{-1} f(x)=\{x\}$. However, in this case it would be possible to map the center of a $3 \times 3$ square to the boundary of it leaving the points of the boundary fixed, obtaining in this way a "continuous" retraction from the square to its boundary, something impossible in the continuous realm. The number of admissible function is reduced considering restrictions, as in its notion of $n$-isomorphism, upon the diameter of the image of a point, although, if we want $f(x)=n x$ to be continuous, these restrictions should be dependent of the function. Nevertheless, this kind of restrictions might be useful, since allowing bigger images we gradually increase the class of allowable functions.

In [14, A. Rosenfeld and A. Nakamura introduce the notion of a local deformation of digital curves that, as they mention, "can be regarded as digitally continuous in the sense that it takes neighboring pixels into neighboring pixels, but it is not exactly the same as a digitally continuous mappings". This is mainly due to the fact that one point in a curve can be related to several points in the other.

The multivalued approach to continuity in digital spaces has also been used by R. Tsaur and M. Smyth in [15], where a notion of continuous multifunction for discrete spaces is introduced: A multifunction is continuous if and only if it is "strong" in the sense of taking neighbors into neighbors with respect to Hausdorff metric. They use this approach to prove some results concerning the existence of fixed points for multifunctions. However, although this approach allows more flexibility in the digitization of continuous functions defined in continuous spaces, it is still a bit restrictive, as shown by the fact that the multivalued function used by them to illustrate the convenience of using multivalued functions is not a strong continuous multifunction.

In this paper, we present a theory of continuity in digital spaces which extends the one introduced by Rosenfeld. In particular, most of the results in [234] are still valid in our context.

In section 1 we revise the basic notions on digital topology required throughout the paper. In particular we recall the different adjacency relations used to model digital spaces. In section 2 we introduce the notion of subdivision of a topological space. This notion is next used to define continuity for multivalued functions and to prove some basic properties concerning the behavior of digitally continuous multivalued functions under restriction and composition. In section 3 we show that the deletion of simple points can be completely characterized in terms of digitally continuous multivalued functions. In particular, a point is simple if and only if certain multivalued function is continuous. Section 4 is devoted to the 
definition and properties of multivalued retractions. We show that the behavior of multivalued retractions in the digital plane is completely analogous to the behavior of retractions in the continuous (real) plane, in contrast with what happens with the existing notions of digital continuity. In the last section we translate to the digital space the well known Hahn-Mazurkiewicz theorem which characterizes locally connected continua as continuous images of the interval.

For information on Digital Topology we recommend the survey [9] and the books by Kong and Rosenfeld [10, and by Klette and Rosenfeld [8].

We are grateful to the referees for their suggestions and remarks which have helped to improve the final version of this paper.

\section{Digital Spaces}

We consider $\mathbb{Z}^{n}$ as model for digital spaces.

Two points in the digital line $\mathbb{Z}$ are adjacent if they are different but their coordinates differ in at most a unit. Two points in the digital plane $\mathbb{Z}^{2}$ are 8adjacent if they are different and their coordinates differ in at most a unit. They are said 4 -adjacent if they are 8 -adjacent and differ in at most a coordinate. Two points of the digital 3-space $\mathbb{Z}^{3}$ are 26-adjacent if they are different and their coordinates differ in at most a unit. They are said 18-adjacent if they are 26 -adjacent and differ in at most two coordinates, and they are said 6-adjacent if they are 26 -adjacent and differ in at most a coordinate. In an analogous way, adjacency relations are defined in $\mathbb{Z}^{n}$ for $n \geq 4$, for example, in $\mathbb{Z}^{4}$ there exist 4 different adjacency relations: 80-adjacency, 64-adjacency, 32-adjacency and 8adjacency.

Given $p \in \mathbb{Z}^{2}$ we define $\mathcal{N}(p)$ as the set of points 8-adjacent to $p$, i.e. $\mathcal{N}(p)=$ $\left\{p_{1}, p_{2}, \ldots, p_{8}\right\}$. This is also denoted as $\mathcal{N}_{8}(p)$. Analogously, $\mathcal{N}_{4}(p)$ is the set of points 4 -adjacent to $p$ (with the above notation $\mathcal{N}_{4}(p)=\left\{p_{2}, p_{4}, p_{6}, p_{8}\right\}$ ).

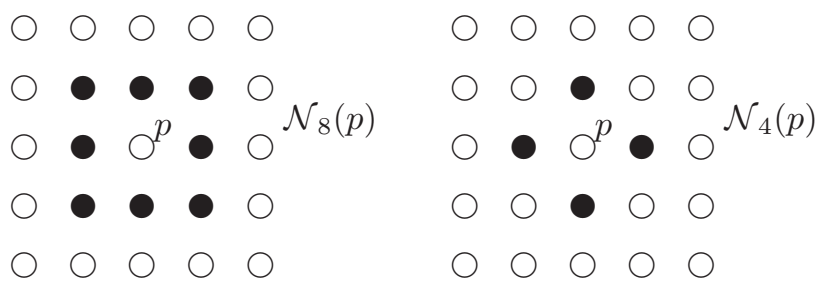

Given $p \in \mathbb{Z}^{3}, \mathcal{N}(p)$ will be the set of points 26-adjacent to $p$. In this case there will exist three kinds of neighborhood: $\mathcal{N}_{26}(p), \mathcal{N}_{18}(p)$ and $\mathcal{N}_{6}(p)$.

A $k$-path $P$ in $\mathbb{Z}^{n}$ (where $k$ corresponds to any of the possible adjacency relations in $\left.\mathbb{Z}^{n}\right)$ is a sequence $P=\left\{p_{0}, p_{1}, p_{2}, \ldots, p_{r}\right\}$ of points such that $p_{i}$ is $k$-adjacent to $p_{i+1}$, for every $i \in\{0,1,2, \ldots, r-1\}$. It is said then that $P$ is a $k$-path from $p_{0}$ to $p_{r}$. If $p_{0}=p_{r}$ then it is called a closed path.

A $k$-path $\left\{p_{0}, p_{1}, p_{2}, \ldots, p_{r}\right\}$ is called a $k$-arc if the only $k$-adjacent points are consecutive points and, possibly, the end points (i.e., if $0 \leq i<i+1<j<r$ or $0<i<i+1<j \leq r$ then $p_{i}$ is not $k$-adjacent to $p_{j}$ ). Every $k$-path $P$ contains a $k$-arc with the same end points. 
A set $S \subset \mathbb{Z}^{n}$ is $k$-connected if for every pair of points of $S$ there exists a $k$-path contained in $S$ joining them. A $k$-connected component of $S$ is a $k$-connected maximal set.

\section{Continuous Multivalued Functions}

Definition 1. Let $f: X \subset \mathbb{Z}^{m} \longrightarrow \mathbb{Z}^{n}$ be a function between digital spaces with adjacency relations $k$ and $k^{\prime}$. According to [13], $f$ is $\left(k, k^{\prime}\right)$-continuous if $f$ sends $k$-adjacent points to $k^{\prime}$-adjacent points. When $m=n$ and $k=k^{\prime}, f$ is said to be just $k$-continuous.

We will say that $f: X \subset \mathbb{Z}^{m} \longrightarrow \mathbb{Z}^{n}$ is continuous if it is $\left(k, k^{\prime}\right)$-continuous for some $k$ and $k^{\prime}$.

In the following definition we introduce the concept of subdivision of $\mathbb{Z}^{n}$.

Definition 2. The first subdivision of $\mathbb{Z}^{n}$ is formed by the set

$$
\mathbb{Z}_{1}^{n}=\left\{\left(\frac{z_{1}}{3}, \frac{z_{2}}{3}, \ldots, \frac{z_{n}}{3}\right) \mid\left(z_{1}, z_{2}, \ldots, z_{n}\right) \in \mathbb{Z}^{n}\right\}
$$

and the $3^{n}: 1$ map $i: \mathbb{Z}_{1}^{n} \hookrightarrow \mathbb{Z}^{n}$ given by $i\left(\frac{z_{1}}{3}, \frac{z_{2}}{3}, \ldots, \frac{z_{n}}{3}\right)=\left(z_{1}^{\prime}, z_{2}^{\prime}, \ldots, z_{n}^{\prime}\right)$ where $\left(z_{1}^{\prime}, z_{2}^{\prime}, \ldots, z_{n}^{\prime}\right)$ is the point in $\mathbb{Z}^{n}$ closer to $\left(\frac{z_{1}}{3}, \frac{z_{2}}{3}, \ldots, \frac{z_{n}}{3}\right)$.

The $r$-th subdivision of $\mathbb{Z}^{n}$ is formed by the set

$$
\mathbb{Z}_{r}^{n}=\left\{\left(\frac{z_{1}}{3^{r}}, \frac{z_{2}}{3^{r}}, \ldots, \frac{z_{n}}{3^{r}}\right) \mid\left(z_{1}, z_{2}, \ldots, z_{n}\right) \in \mathbb{Z}^{n}\right\}
$$

and the $3^{n r}: 1$ map $i_{r}: \mathbb{Z}_{r}^{n} \hookrightarrow \mathbb{Z}^{n}$ given by $i_{r}\left(\frac{z_{1}}{3^{r}}, \frac{z_{2}}{3^{r}}, \ldots, \frac{z_{n}}{3^{r}}\right)=\left(z_{1}^{\prime}, z_{2}^{\prime}, \ldots, z_{n}^{\prime}\right)$ where $\left(z_{1}^{\prime}, z_{2}^{\prime}, \ldots, z_{n}^{\prime}\right)$ is the point in $\mathbb{Z}^{n}$ closer to $\left(\frac{z_{1}}{3^{r}}, \frac{z_{2}}{3^{r}}, \ldots, \frac{z_{n}}{3^{r}}\right)$. Observe that $i_{r}=i \circ i \circ \cdots \circ i$.

Moreover, if we consider in $\mathbb{Z}^{n}$ a $k$-adjacency relation, we can consider in $\mathbb{Z}_{r}^{n}$, in an immediate way, the same adjacency relation, i.e., $\left(\frac{z_{1}}{3^{r}}, \frac{z_{2}}{3^{r}}, \ldots, \frac{z_{n}}{3^{r}}\right)$ is $k$-adjacent to $\left(\frac{z_{1}^{\prime}}{3^{r}}, \frac{z_{2}^{\prime}}{3^{r}}, \ldots, \frac{z_{n}^{\prime}}{3^{r}}\right)$ if and only if $\left(z_{1}, z_{2}, \ldots, z_{n}\right)$ is $k$-adjacent to $\left(z_{1}^{\prime}, z_{2}^{\prime}, \ldots, z_{n}^{\prime}\right)$.

Proposition 1. $i_{r}$ is $k$-continuous as a function between digital spaces.

Definition 3. Given $X \subset \mathbb{Z}^{n}$, the $r$-th subdivision of $X$ is the set $X_{r}=i_{r}^{-1}(X)$. Intuitively, if we consider $X$ made of pixels (resp. voxels), the $r$-th subdivision of $X$ consists in replacing each pixel with $9^{r}$ pixels (resp. $27^{r}$ voxels) and the map $i_{r}$ is the inclusion.

Remark 1. Given $X, Y \subset \mathbb{Z}^{n}$, any function $f: X_{r} \longrightarrow Y$ induces in an immediate way a multivalued function $F: X \longrightarrow Y$ where $F(x)=\bigcup_{x^{\prime} \in i_{r}^{-1}(x)} f\left(x^{\prime}\right)$. 
Definition 4. Consider $X, Y \subset \mathbb{Z}^{n}$. A multivalued function $F: X \longrightarrow Y$ is said to be a $\left(k, k^{\prime}\right)$-continuous multivalued function if it is induced by a $\left(k, k^{\prime}\right)$ continuous (single-valued) function from $X_{r}$ to $Y$ for some $r \in \mathbb{N}$.

Remark 2. Let $F: X \longrightarrow Y\left(X, Y \subset \mathbb{Z}^{n}\right)$ be a $\left(k, k^{\prime}\right)$-continuous multivalued function. Then

i) $F(x)$ is $k^{\prime}$-connected, for every $x \in X$,

ii) if $x$ and $y$ are $k$-adjacent points of $X$, then $F(x)$ and $F(y)$ are $k^{\prime}$-adjacent subsets of $Y$.

iii) $F$ takes $k$-connected sets to $k^{\prime}$-connected sets.

Note that (iii) implies, and is implied by, (i) and (ii).

On the other hand, not all multivalued functions satisfying (i), (ii) and (iii) are induced by continuous single-valued functions. For example, the following multivalued function $F: \mathcal{N}(p) \cup\{p\} \longrightarrow \mathcal{N}(p)$

\begin{tabular}{|c|c|c|}
\hline$p_{1}$ & $p_{2}$ & $p_{3}$ \\
\hline$p_{8}$ & $p$ & $p_{4}$ \\
\hline$p_{7}$ & $p_{6}$ & $p_{5}$ \\
\hline
\end{tabular}

given by

$$
F(x)=\left\{\begin{array}{l}
\mathcal{N}(p) \text { if } x=p \\
\{x\} \text { if } x \in \mathcal{N}(p)
\end{array}\right.
$$

Remark 3. It is immediate that any digitally continuous function in the sense of Rosenfeld is also continuous as a multivalued function. In particular, any single-valued constant map is continuous as a multivalued map.

On the other hand, "strong" continuous multivalued functions in [15] satisfy (ii) above, although the image of a point does not need to be connected. Hence strong multivalued continuity does not imply our notion of continuity. There is even strong continuous multivalued function with the images of all points connected, like the example in the previous remark, which are not continuous as defined in this paper.

Conversely, the function in Example 1 in Section 4 is a continuous multivalued function (as defined here) which is not a strong continuous multivalued function.

The following result is easy to prove.

Proposition 2. If $F: X \longrightarrow Y\left(X, Y \subset \mathbb{Z}^{n}\right)$ is a $\left(k, k^{\prime}\right)$-continuous multivalued function and $X^{\prime} \subset X$ then $\left.F\right|_{X^{\prime}}: X^{\prime} \longrightarrow Y$ is a $\left(k, k^{\prime}\right)$-continuous multivalued function.

If $F: X \longrightarrow Y$ and $G: Y \longrightarrow Z$ are continuous multivalued functions, then we can consider the composition $G F: X \longrightarrow Z$. However, it is not straightforward to prove that $G F$ is continuous. We need first the following result. 
Lemma 1. Let $f: X \longrightarrow Y\left(X \subset \mathbb{Z}^{m}, Y \subset \mathbb{Z}^{n}\right)$ be a $\left(k, k^{\prime}\right)$-continuous function. Then, for every $r \in \mathbb{N}, f$ induces a $\left(k, k^{\prime}\right)$-continuous function $f_{r}$ : $X_{r} \longrightarrow Y_{r}$ such that $f_{r}\left(i_{r}^{-1}(x)\right)=i_{r}^{-1}(f(x))$ for every $x \in X$. This is equivalent to the following diagram

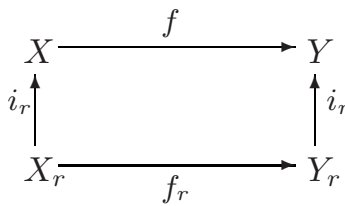

being commutative.

To be precise, we should have denoted by $\left(i_{r}\right)_{X}$ and $\left(i_{r}\right)_{Y}$ the maps $\left(i_{r}\right)_{X}$ : $X_{r} \longrightarrow X$ and $\left(i_{r}\right)_{Y}: Y_{r} \longrightarrow Y$, respectively. In order not to complicate the notation we have denoted both maps by $i_{r}$.

Proof. It is simply an interpolation. For example, when $m=n=2$ and $r=1$, then we have just to define $f_{1}(x, y)=f(x, y)$ if $(x, y) \in \mathbb{Z}^{2}$, while if $(x, y) \notin \mathbb{Z}^{2}$ then $(x, y)=\frac{2}{3}\left(x^{\prime}, y^{\prime}\right)+\frac{1}{3}\left(x^{\prime \prime}, y^{\prime \prime}\right)$ with $\left(x^{\prime}, y^{\prime}\right),\left(x^{\prime \prime}, y^{\prime \prime}\right) \in \mathbb{Z}^{2}$ adjacent points, and we define $f_{1}(x, y)=\frac{2}{3} f\left(x^{\prime}, y^{\prime}\right)+\frac{1}{3} f\left(x^{\prime \prime}, y^{\prime \prime}\right)$.

Theorem 1. If $F: X \longrightarrow Y$ is a $\left(k, k^{\prime}\right)$-continuous multivalued function and $G: Y \longrightarrow Z$ is a $\left(k^{\prime}, k^{\prime \prime}\right)$-continuous multivalued functions, then $G F: X \longrightarrow Z$ is a $\left(k, k^{\prime \prime}\right)$-continuous multivalued function.

Proof. Suppose that $F$ is induced by $f: X_{r} \longrightarrow Y$ and $G$ is induced by $g$ : $Y_{s} \longrightarrow Z$ (observe that $g f$ is not in general well defined).

Consider $X_{r+s}$ the $(r+s)$-subdivision of $X$ which is also the $s$-subdivision of $X_{r}$. Then $f$ induces, according to the above lemma, a $\left(k, k^{\prime}\right)$-continuous function $f_{s}: X_{r+s} \longrightarrow Y_{s}$. Then $g f_{s}: X_{r+s} \longrightarrow Z$ is a $\left(k, k^{\prime \prime}\right)$-continuous function which induces $G F$.

\section{Continuous Multivalued Functions and Simple Points}

It may seem that the family of continuous multivalued functions could be too wide, therefore not having good properties. In this section we show that this is not the case. We show, in particular, that the existence of a $k$-continuous multivalued function from a set $X$ to $X \backslash\{p\}$ which leaves invariant $X \backslash\{p\}$ is closely related to $p$ being a $k$-simple point of $X$.

Let $X \subset \mathbb{Z}^{2}$ and $p \in X$. The point $p$ is called $k$-simple $(k=4,8)$ in $X$ (see [9]) if the number of $k$-connected components of $\mathcal{N}(p) \cap X$ which are $k$-adjacent to $p$ is equal to 1 and $\mathcal{N}_{\bar{k}}(p) \cap X^{c} \neq \emptyset$ (this last condition is equivalent to $p$ being a boundary point of $X$ ). Here $\bar{k}=4$ if $k=8$ and $\bar{k}=8$ if $k=4$.

Theorem 2. Let $X \subset \mathbb{Z}^{2}$ and $p \in X$. Suppose there exists a $k$-continuous multivalued function $F: X \longrightarrow X \backslash\{p\}$ such that $F(x)=\{x\}$ if $x \neq p$ and $F(p) \subset \mathcal{N}(p)$. Then $p$ is a k-simple point. 
The converse is true if and only if $p$ is not 8-interior to $X$ (note that if $p$ is 8-simple it can not be 8-interior, however there are 4-simple points which are 8-interior).

Proof. Suppose that $F$ is induced by $f_{r}: X_{r} \longrightarrow X$. Then $f_{r}(x)=i_{r}(x)$ for every $x \in X_{r}$ such that $i_{r}(x) \neq p$.

Suppose that $p$ is not $k$-simple. We have two possibilities: $p$ is a boundary point with at least two different $k$-connected components of $\mathcal{N}(p) \cap X$ which are $k$-adjacent to $p$, or $p$ is an interior point.

In the first case, let $A$ and $B$ be any two such components. Consider any $x_{r} \in i_{r}^{-1}(p) k$-adjacent to $i_{r}^{-1}(A)$. Then $x=f_{r}\left(x_{r}\right)$ must be $k$-adjacent to $A$ (since $F(A)=A$ ), and since $A$ is a $k$-connected component of $\mathcal{N}(p) \cap X$, then $f_{r}\left(x_{r}\right) \in A$. On the other hand, there exists also $y_{r} \in i_{r}^{-1}(p) k$-adjacent to $i_{r}^{-1}(B)$ and, hence, $f_{r}\left(y_{r}\right)=y \in B$. Consider $\left\{z_{0}=x_{r}, z_{1}, z_{2} \ldots, z_{m-1}, z_{m}=\right.$ $\left.y_{r}\right\} \subset i_{r}^{-1}(p)$ such that $z_{i}$ is $k$-adjacent to $z_{i-1}$ for every $i=1,2, \ldots, m$. Then $\left\{f_{r}\left(z_{0}\right)=x, f_{r}\left(z_{1}\right), f_{r}\left(z_{2}\right), \ldots, f_{r}\left(z_{m-1}\right), f_{r}\left(z_{m}\right)=y\right\} \subset \mathcal{N}(p) \cap X$ is a $k$-path in $\mathcal{N}(p) \cap X$ from $x$ to $y$. Contradiction.

Suppose now that $p$ is an interior point and that there exists a $k$-continuous multivalued function $F: X \longrightarrow X \backslash\{p\}$ such that $F\left(p_{i}\right)=\left\{p_{i}\right\}$ for every $i=1,2, \ldots, 8$. Consider a subdivision $X_{r}$ of $X$ and a $k$-continuous map $f_{r}$ : $X_{r} \longrightarrow X$ which induces $F$.

We divide $i_{r}^{-1}(p)$ into concentric paths (the first path would be its boundary, the next path would be the boundary of the interior, and so on). Then, by continuity, for the outer path, there are points in it whose images are $p_{2}, p_{4}, p_{6}$ and $p_{8}$. Therefore $F(p) \subset\left\{p_{2}, p_{4}, p_{6}, p_{8}\right\}$. If we consider now the next concentric paths of $i_{r}^{-1}(p)$, by the continuity of $f_{r}$, in any of them there are points whose images are $p_{2}, p_{4}, p_{6}$ and $p_{8}$. In particular, this will hold for the innermost path, making impossible to define $f_{r}$ for the point in the center in a consistent way.

To prove the converse statement, consider first the following situation, with $p \in X$ a $k$-simple point such that $\mathcal{N}(p) \cap X=\left\{p_{1}, p_{3}, p_{4}, p_{5}, p_{6}, p_{7}, p_{8}\right\}$.

\begin{tabular}{|l|l|l|}
\hline$p_{1}$ & & $p_{3}$ \\
\hline$p_{8}$ & $p$ & $p_{4}$ \\
\hline$p_{7}$ & $p_{6}$ & $p_{5}$ \\
\hline
\end{tabular}

We are going to construct $F$ as in the statement of the theorem. To do that we consider the second subdivision $X_{2}$ of $X$ and we are going to construct a singlevalued function $f: X_{2} \longrightarrow X$ which induces the desired $F$. Since $F(x)=\{x\}$ for every $x \in \mathcal{N}(p) \cap X$, then $f\left(x^{\prime}\right)=x$ for every $x^{\prime} \in i_{2}^{-1}(x)$. In order to define $f\left(p^{\prime}\right)$ for $p^{\prime} \in i_{2}^{-1}(p)$ we divide $i_{2}^{-1}(p)$ in groups and define $f$ as shown by the arrows in the following figure. 


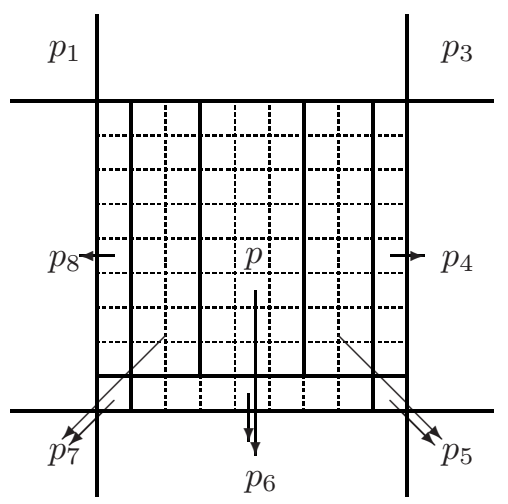

Then $f: X_{2} \longrightarrow X$ is 4-continuous and 8-continuous. Therefore, if we define $F(p)=\left\{p_{4}, p_{5}, p_{6}, p_{7}, p_{8}\right\}, F$ is a 8 -continuous and 4-continuous multivalued function.

If $p$ is any other simple point in the hypothesis of the theorem then there exists a point in $\mathcal{N}_{4}(p)$ which is not in $X$ but one (or both) of the (clockwise) next points in $\mathcal{N}(p)$ are in $X$. We may suppose, making, if necessary, a rotation of $X$ (by 90,180 or 270 degrees) that $p_{2}$ is that point.

The same groups in $i_{2}^{-1}(p)$ can be used to define $F(p)$ in any of these cases. To show it, we label these groups as follows

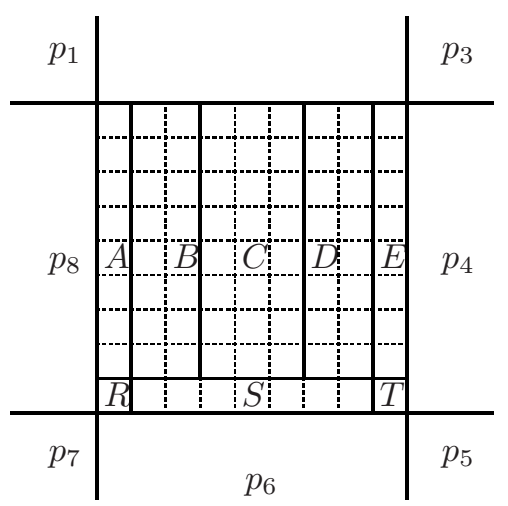

There are two possibilities: either $\mathcal{N}(p) \cap X=\left\{p_{3}\right\}$, in which case we define $F(p)=$ $\left\{p_{3}\right\}$, or $p_{4} \in X$. In the second case we have again two possibilities: either $p_{6} \notin X$, in which case $\mathcal{N}(p) \cap X \subset\left\{p_{3}, p_{4}, p_{5}\right\}$ and we define $F(p)=\left\{p_{4}\right\}$, or $\left\{p_{4}, p_{6}\right\} \subset X$. If $\left\{p_{4}, p_{6}\right\} \subset X$ but $p_{8} \notin X$, then $\mathcal{N}(p) \cap X \subset\left\{p_{3}, p_{4}, p_{5}, p_{6}, p_{7}\right\}$. If $p_{5} \notin X$ we define $F(E \cup T)=p_{4}$ and $F(A \cup B \cup C \cup D \cup R \cup S)=p_{6}$, while if $p_{5} \in X$ we define $F(E)=p_{4}, F(D \cup T)=p_{5}$ and $F(A \cup B \cup C \cup R \cup S)=p_{6}$.

Finally, if $\left\{p_{4}, p_{6}, p_{8}\right\} \subset X$, we consider two cases. If $\left\{p_{5}, p_{7}\right\} \subset X$, we define $F(E)=p_{4}, F(D \cup T)=p_{5}, F(C \cup S)=p_{6}, F(B \cup R)=p_{7}$ and $F(A)=p_{8}$. On 
the other hand, if $\left\{p_{5}, p_{7}\right\} \not \subset X$, then $p$ can only be 8-simple (not 4-simple), and we can define $F(D \cup E \cup T)=p_{4}, F(C \cup S)=p_{6}, F(A \cup B \cup R)=p_{8}$.

We see finally that if $p$ is 4-simple and 8-interior, then there is not a 4continuous multivalued function $F: X \longrightarrow X \backslash\{p\}$ such that $F(x)=\{x\}$ if $x \neq p$ and $F(p) \subset \mathcal{N}(p)$. To see this, observe first that $\mathcal{N}(p) \cap X$ must be as follows (or a rotation of it)

\begin{tabular}{|l|l|l|}
\hline$p_{1}$ & $p_{2}$ & \\
\hline$p_{8}$ & $p$ & $p_{4}$ \\
\hline$p_{7}$ & $p_{6}$ & $p_{5}$ \\
\hline
\end{tabular}

Suppose there exists a 4-continuous multivalued function $F: X \longrightarrow X \backslash\{p\}$ such that $F(x)=\{x\}$ if $x \neq p$ and $F(p) \subset \mathcal{N}(p) \cap X$. Suppose that $F$ is induced by $f_{r}: X_{r} \longrightarrow X$. Then $f_{r}(x)=p_{2}$ for every $x \in X_{r}$ such that $i_{r}(x)=p_{2}$ and $f_{r}(x)=p_{4}$ for every $x \in X_{r}$ such that $i_{r}(x)=p_{4}$. Then if we consider the upper rightmost point $x \in i_{r}^{-1}(p)$, it is not possible to define $f_{r}(x)$ in such a way that $f_{r}$ is 4-continuous.

Remark 4. $F$ is not unique. For example, for $\mathcal{N}(p) \cap X$ as follows,

\begin{tabular}{|l|l|l|}
\hline$p_{1}$ & & $p_{3}$ \\
\hline$p_{8}$ & $p$ & $p_{4}$ \\
\hline$p_{7}$ & $p_{6}$ & $p_{5}$ \\
\hline
\end{tabular}

if we define $F(p)=\left\{p_{1}, p_{3}, p_{4}, p_{5}, p_{6}, p_{7}, p_{8}\right\}$, then $F$ is still a 8 -continuous and 4-continuous multivalued function. The same is true if we define $F(p)=$ $\left\{p_{3}, p_{4}, p_{5}, p_{6}, p_{7}, p_{8}\right\}$. However, if we define $F(p)=\left\{p_{5}, p_{6}, p_{7}, p_{8}\right\}$, then $F$ is nor a 8-continuous neither a 4-continuous multivalued function, since (ii) in Remark 2 does not hold in this case $\left(p_{3}\right.$ and $p$ are adjacent points of $X$, but $F\left(p_{3}\right)$ and $F(p)$ are not adjacent subsets of $X)$.

Remark 5. It is easy to see that for $\mathcal{N}(p) \cap X$ as in Remark 4, although $p$ is 4-simple and 8-simple, any single-valued function $f: X \longrightarrow X \backslash\{p\}$, such that $f(x)=x$ if $x \neq p$, can not be 4-continuous neither 8-continuous, hence Theorema 2 does not hold for Rosenfeld's digitally continuous functions.

Remark 6. There exist in the literature results characterizing simple points in terms of properties of certain inclusion maps, in the spirit of our theorem. For example, in [12] (see [56] for further and more recent results) simple surfels (the equivalent for a digital surface $X$ of simple points in the digital plane) are characterized as points $x$ such that the morphism $i_{*}: \Pi_{1}^{n}(X \backslash x) \longrightarrow \Pi_{1}^{n}(X)$ is an isomorphism. 


\section{Multivalued Digital Retractions}

In the previous section we have characterized the deletion of a $k$-simple point in $\mathbb{Z}^{2}$ as a $k$-continuous multivalued function $f: X \longrightarrow X \backslash\{p\}$ such that $f(x)=x$ if $x \neq p$. This is a particular case of a wider class of functions known as multivalued retractions.

Definition 5. Consider $Y \subset X \subset \mathbb{Z}^{n}$. A multivalued $k$-retraction from $X$ to $Y$ is a $k$-continuous multivalued function $F: X \longrightarrow Y$ such that $f(y)=\{y\}$ for every $y \in Y$.

Remark 7. If we look at the notion of simple connectivity preserving mapping [11, it is clear that if a connectivity preserving mapping from $X$ to a subset $Y$ has to leave $Y$ fixed, then if it is simple it must be single-valued. Therefore, in that case, retractions would agree with those in [1].

The next two results are the digital versions of two well known facts about $\mathbb{R}^{2}$, namely, that the boundary of a disk is not a retract of the whole disk, while an annulus (or of a punctured disk) can be retracted to its outer boundary.

Proposition 3. The boundary $\partial X$ of a square $X$ is not a $k$-retract of $X(k=$ $4,8)$.

Proof. Let $p_{N}, p_{E}, p_{S}, p_{W}$ be points in each of the four sides of $\partial X$, different from the corner points. Suppose that there exists a $k$-continuous multivalued function $F: X \longrightarrow \partial X$ such that $F(p)=\{p\}$ for every $p \in \partial X$. Consider a subdivision $X_{r}$ of $X$ and a $k$-continuous map $f_{r}: X_{r} \longrightarrow X$ which induces $F$.

Divide $i_{r}^{-1}(X)$ into concentric paths. Then, by continuity, for the outer path in $X \backslash \partial X$, there are points in it whose images by $f_{r}$ are, respectively $p_{N}, p_{E}$, $p_{S}$ and $p_{W}$. If we successively consider the different concentric paths of $i_{r}^{-1}(p)$, by the continuity of $f_{r}$, in any of them there are points whose images are $p_{N}$, $p_{E}, p_{S}$ and $p_{W}$. In particular, this will hold for the innermost path, making it impossible to define $f_{r}$ for the point at the center in a consistent way.

Example 1. Let $X$ be a squared annulus and consider $F: X \longrightarrow X$ defined as follows

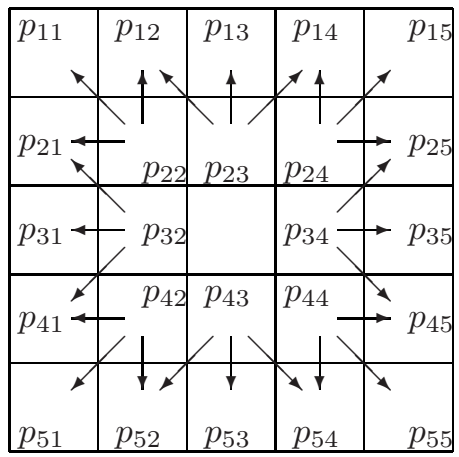


i.e. $F$ is the identity on the outer boundary of the annulus, and for every $p$ in the inner boundary, $F(p)$ is equal to three points in the outer boundary.

Then $F$ is a multivalued $k$-retraction for $k=4$ and $k=8$.

Proof. $F$ is induced by the following map $f_{1}: X_{1} \longrightarrow X$ :

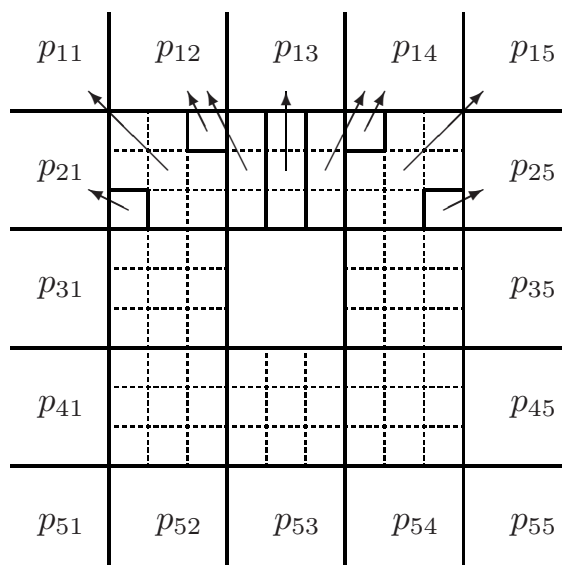

where the rest of the images are completed symmetrically.

This result improves [1, Theorem 4.4]. There, it was proved that the boundary of a filled square is not a retract of the whole square, but the same arguments in the proof of that theorem shows that neither is the boundary of a squared annulus a digital retract (according to Boxer's definition) of it.

\section{Digital Version of Hahn-Mazurkiewicz Theorem}

By the well-known Hahn-Mazurkiewicz theorem (see, for example, [7]), any locally connected continuum (compact and connected set) is the continuum image of the interval $[0,1]$ and, conversely, any continuum image of the interval $[0,1]$ is a locally connected continuum. We end the paper with a digital version of this result.

Theorem 3 (Digital version of Hahn-Mazurkiewicz theorem). Let $X \subset$ $\mathbb{Z}^{n}$. Consider $I=\{0,1\}$ the digitalunitinterval. Then, there exists a surjective $(2, k)$ continuous multivalued function $F: I \longrightarrow X$ if and only $X$ is a bounded $k$-connected set (where $k$ correspond to any of the possible adjacency relations in $\mathbb{Z}^{n}$ ).

Proof. Suppose there exists a surjective $(2, k)$-continuous multivalued function $F: I \longrightarrow X$. Since any subdivision of $I$ is a finite 2-connected set, then $X=F(I)$ must be finite and hence bounded and also $k$-connected (by Remark 2).

Suppose, on the other hand, that $X$ is a bounded $k$-connected set. Then, since it is also finite, there exists a $k$-path $P=\left\{p_{0}, p_{1}, p_{2}, \ldots, p_{n}\right\}$ which goes through all the points in $X$ (the path may go more than once through each point). Consider $I_{r}$ subdivision of $I$ such that $2 \cdot 3^{r} \geq n+1$. Then there exists a $k$-continuous function $f: I_{r} \longrightarrow P$ which induces a $k$-continuous multivalued function $F: I \longrightarrow X$. 
Remark 8. In the general version of Hahn-Mazurkiewicz theorem, $X$ is required to be compact and locally connected. These conditions do not appear in the digital version because all bounded digital sets satisfy these two properties.

Remark 9. The digital version of Hahn-Mazurkiewicz theorem can be stated in a more surprising way as follows: $X$ is a bounded $k$-connected set if and only $F:\{p\} \longrightarrow X$ given by $F(p)=X$ is a $k$-continuous multivalued function. This is a consequence of the existence of a $k$-continuous multivalued function $F:\{p\} \longrightarrow I$ defined in an obvious way.

As a consequence, since a single-valued constant map is continuous (as a multivalued function), then, for every digital sets $X$ and $Y$ ( $Y k$-connected and bounded), the map $F: X \longrightarrow Y$, given by $F(p)=Y$ for every $p \in X$, is a surjective $k$-continuous multivalued function.

\section{References}

1. Boxer, L.: Digitally continuous functions. Pattern Recognition Letters 15, 833-839 (1994)

2. Boxer, L.: A Classical Construction for the Digital Fundamental Group. Journal of Mathematical Imaging and Vision 10, 51-62 (1999)

3. Boxer, L.: Properties of Digital Homotopy. Journal of Mathematical Imaging and Vision 22, 19-26 (2005)

4. Boxer, L.: Homotopy properties of Sphere-Like Digital Images. Journal of Mathematical Imaging and Vision 24, 167-175 (2006)

5. Burguet, J., Malgouyres, R.: Strong thinning and polyhedric approximation of the surface of a voxel object. Discrete Applied Mathematics 125, 93-114 (2003)

6. Fourey, S., Malgouyres, R.: Intersection number and topology preservation within digital surfaces. Theoretical Computer Science 283, 109-150 (2002)

7. Hocking, J.G., Young, G.S.: Topology. Addison-Wesley, Reading (1961)

8. Klette, R., Rosenfeld, A.: Digital Geometry. Elsevier, Amsterdam (2004)

9. Kong, T.Y., Rosenfeld, A.: Digital Topology: Introduction and survey. Computer Vision, Graphics and Image Processing 48, 357-393 (1989)

10. Kong, T.Y., Rosenfeld, A. (eds.): Topological algorithms for digital image processing. Elsevier, Amsterdam (1996)

11. Kovalevsky, V.: A new concept for digital geometry. In: Ying-Lie, O., et al. (eds.) Shape in Picture. Proc. of the NATO Advanced Research Workshop, Driebergen, The Netherlands (1992), Computer and Systems Sciences, vol. 126. Springer-Verlag (1994)

12. Malgouyres, R., Lenoir, A.: Topology preservation within digital surfaces. Comput. Graphics Image Process. Mach. Graphics Vision 7, 417-426 (1998)

13. Rosenfeld, A.: Continuous functions in digital pictures. Pattern Recognition Letters 4, 177-184 (1986)

14. Rosenfeld, A., Nakamurab, A.: Local deformations of digital curves. Pattern Recognition Letters 18, 613-620 (1997)

15. Tsaur, R., Smyth, M.B.: Continuous multifunctions in discrete spaces with applications to fixed point theory. In: Bertrand, G., Imiya, A., Klette, R. (eds.) Digital and Image Geometry, Dagstuhl Seminar 2000. LNCS, vol. 2243, pp. 75-88. Springer, Heidelberg (2002) 